\title{
Recurrent erythema annulare centrifugum and tonsillitis
}

\author{
sana bouzid ${ }^{1}$, Mouna Korbi ${ }^{1}$, khaoula ameur ${ }^{1}$, Hichem Belhadjali ${ }^{1}$, Monia Youssef ${ }^{1}$, and \\ Jameleddine Zili ${ }^{1}$ \\ ${ }^{1}$ Fattouma Bourguiba University Hospital of Monastir
}

March 6, 2022

\begin{abstract}
Recurrent post-tonsillitis erythema annulare centrifugum is a recently described entity in two adults with recurrent annular lesions and centrifugal evolution following tonsillitis. We present two cases of recurrent post-tonsillitis erythema annulare centrifugum in children.
\end{abstract}

Title page:

Recurrent erythema annulare centrifugum and tonsillitis

Authors: Bouzid Sana, Korbi Mouna, AmeurKhaoula, BelhadjaliHichem, Youssef Monia, ZiliJameleddine

Institution: Dermatology Department, University Hospital of Monastir,University of medicine, University of Monastir, Tunisia

Address: Dermatology Department, University Hospital of Monastir, University of medicine, University of Monastir, 5000 Monastir, Tunisia

Corresponding author : Dr Bouzid Sana, address: Dermatology Department, University Hospital of Monastir, 5000 Monastir, Tunisia;

Email:Bouzidsana18@gmail.com

Conflict of interest: None

Written informed consent was obtained from the patient to publish this report in accordance with the journal's patient consent policy

\section{Manuscript:}

Erythema annulare centrifugum (EAC) is a gyrate erythema, which is classically characterized by annular, erythematous plaques with central clearing and a trailing scale. Although its physiopathology remains unclear, a hypersensitivity reaction to various external or internal stimuli has been postulated. Recurrent forms of EAC are regarded as peculiar clinical variants with intriguing precipitating factors [1]. Recently, two cases of recurrent post tonsillitis EAC in adults have been reported in literature. Herein, we present two rare cases of children developing recurrent EAC following tonsillitis.

Case 1:

A 3-year-old boy presented to our Dermatology Department for a two-month-history of recurrent asymptomatic skin lesions. There was no history of potential trigger factors, such as drugs or tick bite. Physical examination revealed multiple erythematous annular and urticariform plaques, with a central clearing and non-scaling borders, located on his face, his right limb and thighs (Fig1). Oto-Rhino-Laryngological (ORL) 
examination revealed erythematous tonsillitis. No mucosal lesions were present and the rest of the physical examination disclosed no abnormalities. Hematologic (complete blood count, sedimentation rate), biochemical (C-reactive protein) and immunologic (anti-nuclear antibodies and anti-DNA antibodies) investigations were within normal levels except for a high rate of antistreptolysin O (ASLO). Careful questioning revealed that relapses were always preceded by tonsillitis. Cutaneous lesions healed completely after receiving adequate antibiotics and the child had no relapses after one-year follow-up.

\section{Case 2:}

A 4-year-old girl presented with a four-month history of recurrent self-healing erythematous plaques without fever or itch. These lesions had annular disposition and polycyclic borders and were located on her lower limbs (Fig2). Clinical examination revealed an erythematous tonsillitis and no cardiac or osteoarticular involvement. The patient's mother stated that tonsillitis seemed to precede the skin lesions on every recurrence. Blood investigations were non-contributory except for a high rate of ASLO. Remission was obtained after treating the tonsillitis and there were no relapses observed after a 4-month-follow-up.

EAC is considered to be a reactive process with a self-limited course and good prognosis. Multiple inciting factors were reported in the literature, mainly infectious such Epstein-Barr virus, dermatophytes, Escherichia coli and recently streptococcal infection. Recurrent post tonsilitis EAC is a controversial entity described by Elfatoiki et al in two women, aged respectively 25 and 33 years, who presented with relapsing annular erythema following streptococcal throat infection [2]. The authors had performed a skin biopsy for their patients revealing non-specific inflammation suggestive of a superficial form of EAC [2]. For our two cases, we did not perform skin biopsy as the clinical presentation was suggestive and the evolution was rapidly regressive. The differential diagnoses of EAC include annular urticaria, Sweet syndrome, lupus and other figurate erythema such as erythema marginatum or erythema migrans [2]. Annular urticaria was not retained in our two patients because the eruption was non itchy and a prolonged centrifugal evolution was noted. Lyme disease, Sweet syndrome and lupus were ruled out based on clinical and biological features. Relapsing annular erythema following streptococcal throat infection with a high rate of ASLO are highly suggestive of erythema marginatum, one of the main Jones diagnostic criteria for rheumatic fever. Indeed, according to Elfatoiki et al, the absence of: arthralgia, cardiac involvement and biological inflammatory syndrome, suggests the diagnosis of this recent entity [2].

Although streptococcal superantigens are widely known to play major roles in reactive processes, the connection between throat streptococcal infections and EAC is yet to be

established. Thus, we believe that accumulation of additional similar cases is required to clarify the exact nature of this association.

\section{References:}

[1] García Muret MP, Pujol RM, Gimenez-Arnau AM, et al. Annually recurring erythema annulare centrifugum: a distinct entity?. J Am Acad Dermatol 2006;54:1091-1095.

[2] Elfatoiki F-Z, Chiheb S, Marnissi S, et al. Recurrent post-tonsillitis erythema annulare centrifugum: two cases. Ann Dermatol Venereol2014;141:219-220. doi:10.1016/j. 


$$
\text { 圆 }
$$




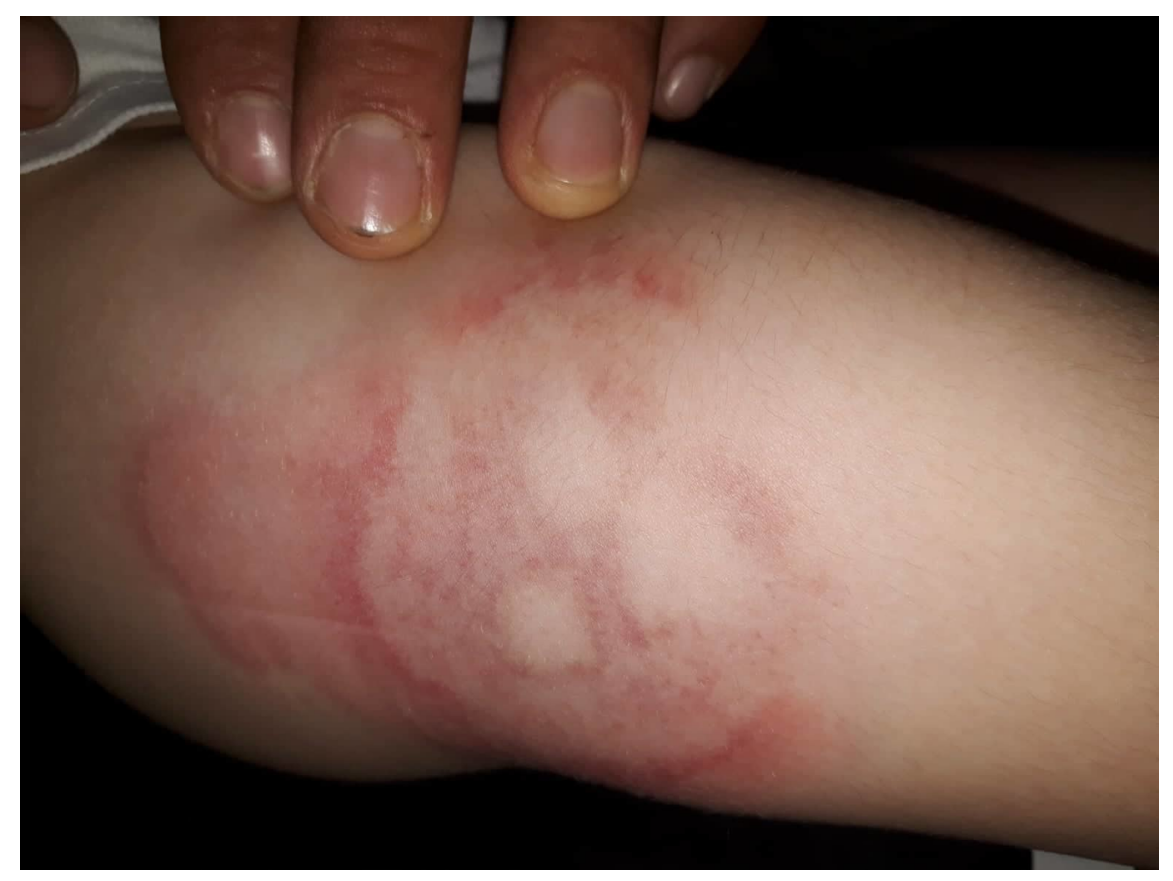

